\title{
Synthesis, structure, and antimicrobial activity of heterocyclic phenylsulfonyl- and 4-aminophenylsulfonyl-carboximidamides
}

\author{
Katarzyna Gobis $\cdot$ Henryk Foks $\cdot$ Katarzyna Wiśniewska • \\ Maria Dąbrowska-Szponar • Ewa Augustynowicz-Kopeć • \\ Agnieszka Napiórkowska $\cdot$ Artur Sikorski
}

Received: 21 January 2012/ Accepted: 11 April 2012/Published online: 15 May 2012

(C) The Author(s) 2012. This article is published with open access at Springerlink.com

\begin{abstract}
A series of novel phenylsulfonyl- and 4-aminophenylsulfonyl-carboximidamides were synthesized by condensation of sulfonamides with heterocyclic methyl carbimidates obtained from heterocyclic carbonitriles and used 'at its inception.' The molecular structure of the obtained compounds is discussed. Compounds possessing heterocyclic systems with a nitrogen atom in the $\alpha$ position to the functional group showed a different single-crystal structure than expected. The synthesized derivatives were evaluated for antimicrobial activities: tuberculostatic, antibacterial, and antifungal.
\end{abstract}

Keywords Sulfonamidine $\cdot$ Heterocycles $\cdot$ Synthesis · Crystal structure - Antimicrobial activity ·

Structure-activity relationship

\section{Introduction}

At the end of the twentieth century, a number of new and "reemerging" pathogens were recognized [1]. These

K. Gobis $(\bowtie) \cdot$ H. Foks

Department of Organic Chemistry, Medical University

of Gdańsk, Gdańsk, Poland

e-mail: kgobis@gumed.edu.pl

K. Wiśniewska · M. Dąbrowska-Szponar Department of Medicinal Microbiology, Chair of Microbiology, Medical University of Gdańsk, Gdańsk, Poland

E. Augustynowicz-Kopeć · A. Napiórkowska

Department of Microbiology, Institute of Tuberculosis and Pulmonary Diseases, Warsaw, Poland

A. Sikorski

Department of Physical Chemistry, University of Gdańsk, Gdańsk, Poland included S. pneumonia, L. pneumophila, M. avium, E. coli, H. pylori, S. aureus, C. albicans, and M. tuberculosis [2-4]. These microorganisms quickly develop a multidrug resistance (MDR) to used chemotherapeutics and antibiotics. A special case is M. tuberculosis, whose strains also develop extensive drug-resistance (XDR). Resistant strains of microorganisms are a major threat to immunocompromised individuals, and infections caused by them are the most common complication in HIV-positive patients [5]. At the same time, a lack of development of new antimicrobial drugs is observed, which can pose a serious threat to public health [6]. Thus, the interest of many research groups is focused on the search for new drugs active against resistant strains.

One of the research directions is to modify the structure of already used drugs. So the interest in chemical groups such as, for example, sulfonamides has not diminished. This group is characterized by multidirectional pharmacological activity. Sulfonamides act as anhydrase inhibitors [7], antifungal [8], antiviral [9], anticancer [10], antiinflammatory [11], and of course antibacterial agents.

Multidirectional biological activity also characterizes compounds possessing an amidine functional group. Amidine derivatives have anti-degenerative [12], antitumor [13], and anti-platelet effects [14]. Compounds with anti$\mathrm{HIV}$, antibacterial, and antifungal activities have also been found among them $[15,16]$.

There are few reports on the pharmacological activity of sulfonamidines. So far, only their in vitro ability to compete with triiodothyronine for binding to the thyroid hormone- $\alpha 1$ receptor (hTHR- $\alpha 1$ ) has been described [17]. These compounds can be obtained in several ways. They are formed as a result of the reaction of carbonitriles with primary sulfonamides [18] or in a reaction of amidines with sulfonyl chlorides [19]. The reports of reactions of 
sulfonamides with alkyl- or phenylcarbimidates could also be found in the chemical literature [20]. In the structure assigned to the products, two protons are connected to different nitrogen atoms of the amidine moiety [21]. That structure was adopted on the basis of ${ }^{1} \mathrm{H}$ NMR spectra in which two different signals for those protons were observed. The reaction of sulfonamides with heterocyclic carbimidates has not been described so far.

The above facts prompt us to synthesize sulfonyl-carboximidamides possessing in their structure phenylsulfonyl or 4-aminophenylsulfonyl moieties linked to heterocyclic rings of pyridine, pyrimidine, or pyrazine by the sulfonamidine group. Synthesized compounds have been evaluated for their antimicrobial activity in vitro: tuberculostatic, antibacterial, and antifungal.

\section{Results and discussion}

The subject of this work was the synthesis of heterocyclic phenylsulfonyl- and 4-aminophenylsulfonyl-carboximidamides 1-13. The performed reactions are shown in Scheme 1.
The presented method of synthesis uses an intermediate such as carbimidate "at its inception," and this is its main advantage. Carbimidates were obtained from the corresponding carbonitriles in methanol in the presence of 1,8diazabicyclo[5.4.0]undec-7-ene (DBU) and without isolation underwent further reaction with benzene sulfonamide or 4-aminobenzenesulfonamide. The isolated carbimidates were used for the synthesis of pyrazine $(\mathbf{1 0}, \mathbf{1 1})$ and 6-methoxypyrazine $(\mathbf{1 2}, \mathbf{1 3})$ derivatives. They were obtained easily from 2-cyanopyrazine and 6-chloro-2-cyanopyrazine, respectively [22, 23]. Carbimidates were refluxed with benzene sulfonamide or 4-aminobenzenesulfonamide in diglyme (2-methoxyethyl ether) solution. All reactions proceeded with yields from moderate $(38 \%)$ to very good $(83 \%)$.

The structures of all these new compounds were confirmed by IR and NMR spectra as well as elemental analyses. Two signals for the NH groups shifted from each other have been observed in the ${ }^{1} \mathrm{H}$ NMR spectra. These separated signals can be due to the taken amino-imine structure of compounds obtained (Fig. 1, structure a) as we suggest for 3- and 4-pyridine derivatives 3-6. They can

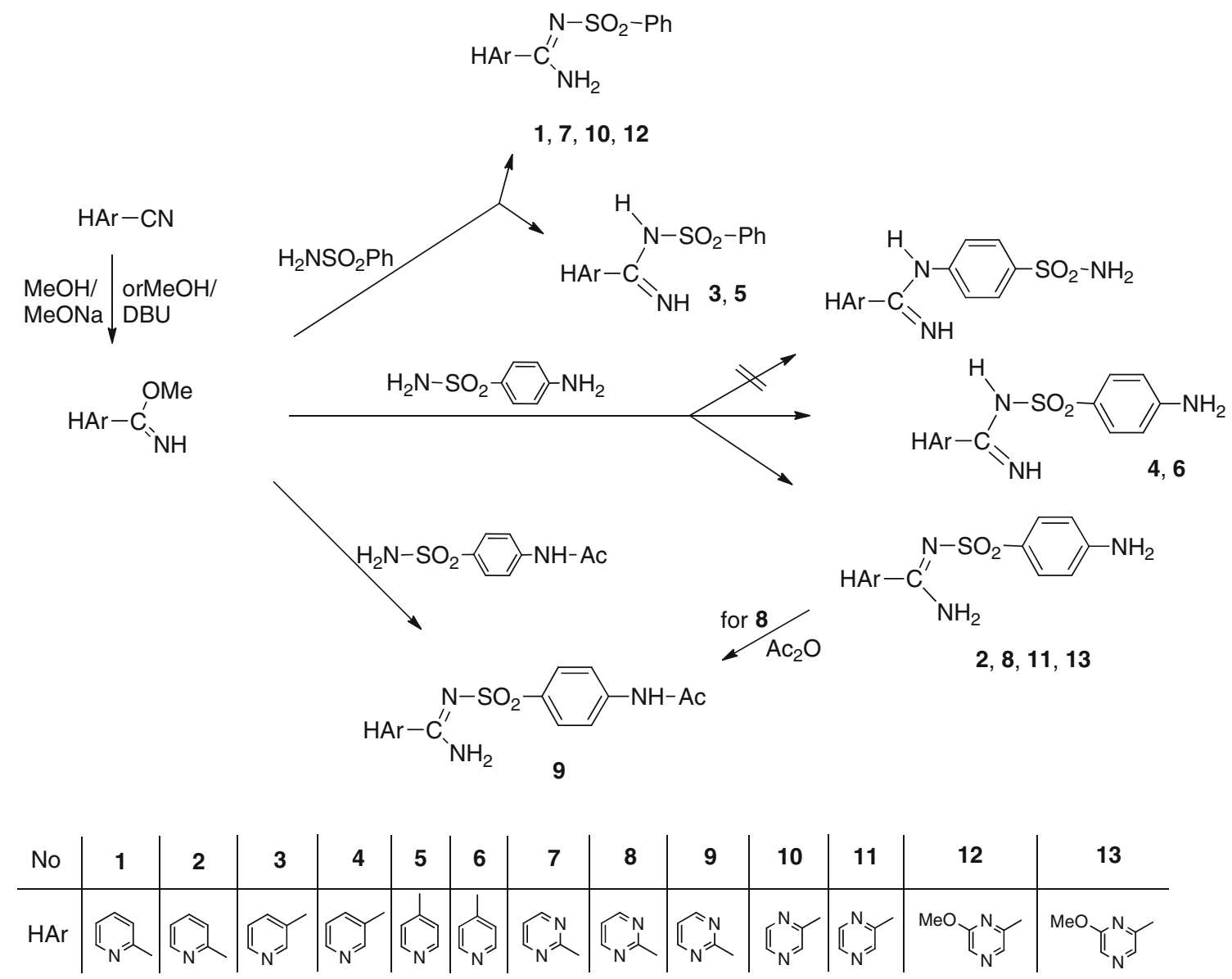

Scheme 1 
<smiles>O=S1(=O)N=C(C2=NCCCC2)N=CN1</smiles>

A<smiles></smiles>

B
Fig. 1 Possible systems of intramolecular hydrogen bonds in target molecules

also be a result of the magnetic inequivalence of $\mathrm{NH}$ protons in the amine moiety upon formation of a hydrogen bond in the case of the heterocyclic compounds in which the amidine group is in the $\alpha$ position to the nitrogen atom of heterocyclic ring (structure b). X-ray diffraction analysis was performed for $N^{\prime}$-(4-aminophenylsulfonyl)-4-chloropicolinimidamide to address that question. We have described the synthesis of this compound previously [24]. It was chosen because we were able to obtain its crystals of sufficient size. The results of the single-crystal diffraction study confirmed a tautomeric structure b (Fig. 1). If both hydrogen atoms are bonded to the same nitrogen atom in the solid state, their magnetic inequivalence in the solution is probably caused by formation of hydrogen bonds and reduction of symmetry.

The study also resolved the molecular structure of the products of the reaction between carbimidates and 4-aminobenzenesulfonamide, as the active group in that reaction could be both the amine group of the sulfonamide moiety, as it was in the case of the reaction of benzene sulfonamide, or the aromatic amine group in the para position to the sulfonamide moiety, since the reactions of aromatic and aliphatic amines with carbimidates have been described [25, 26]. For that purpose, the reaction of methyl pyrazine-2-carbimidate with $N$-(4-sulfamoylphenyl)acetamide was carried out. The resulting product 9 was identical with the compound that was obtained by the acetic anhydride acylation of derivative $\mathbf{8}$, which was formed in the reaction of methyl pyrazine-2carbimidate with 4-aminobenzenesulfonamide. This showed that the sulfonamide group was the active group in the reactions carried out, and the resulting compounds had structure c (Fig. 2).

\section{Crystal structure of $N^{\prime}$-(4-aminophenylsulfonyl)-4- chloropicolinimidamide}

The crystallographic data, data collection, and structure refinement of $N^{\prime}$-(4-aminophenylsulfonyl)-4-chloropicolinimidamide are summarized in Table 1. The bond lengths and angles characterizing the geometry of the molecules are presented in Table 2.

$N^{\prime}$-(4-Aminophenylsulfonyl)-4-chloropicolinimidamide crystallized in the $\mathrm{P} 2{ }_{1} / \mathrm{c}$ monoclinic space group, with
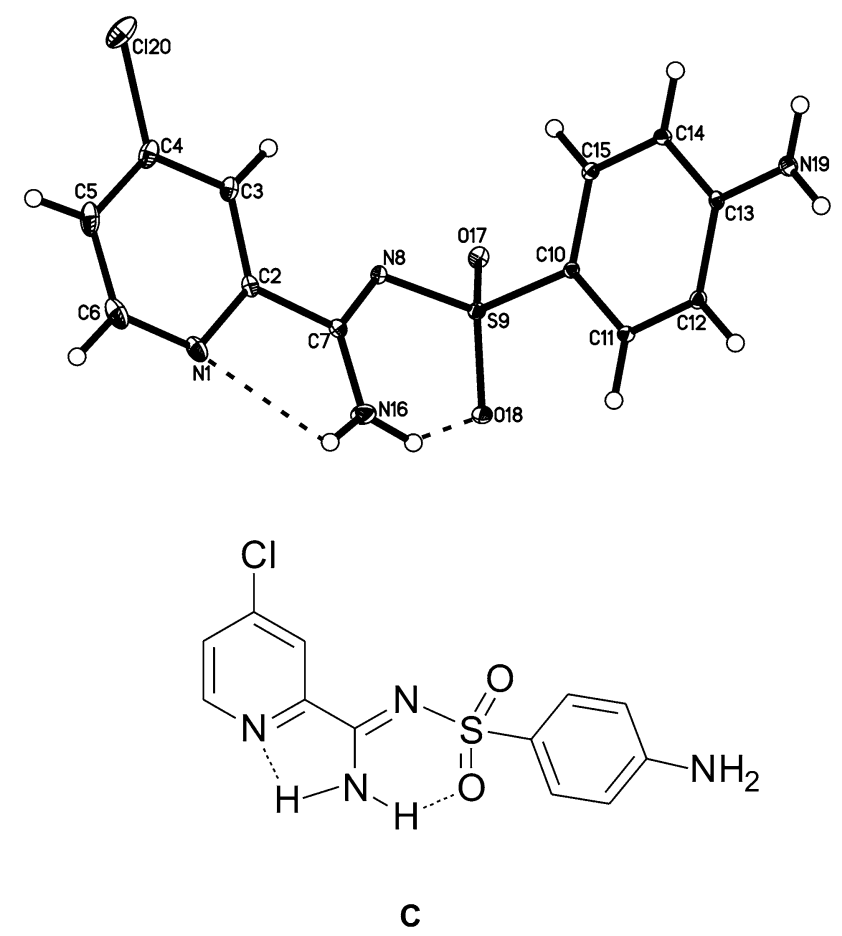

Fig. 2 Structure of $N^{\prime}$-(4-aminophenylsulfonyl)-4-chloropicolinimidamide showing $25 \%$ probability displacements for ellipsoids. $\mathrm{H}$ atoms are shown as small spheres of arbitrary radius (intramolecular $\mathrm{N}-\mathrm{H} \cdots \mathrm{O}$ and $\mathrm{N}-\mathrm{H} \cdots \mathrm{N}$ interactions are represented by dashed lines)

$a=14.6885(7) \AA, \quad b=5.7930(3) \AA, \quad c=16.0421(9) \AA$, and $\beta=97.530(5)^{\circ}, Z=4$, and $V=1353.25(12) \AA^{3}$.

In the molecule of the title compound (Fig. 2), the bond lengths and angles characterizing the geometry of the 4-aminophenylsulfonyl and pyridine fragments are typical for compounds containing them (Table 2).

In the crystal structure of $N^{\prime}$-(4-aminophenylsulfonyl)-4chloropicolinimidamide, the $\mathrm{H}$ atoms from the amino group bonded with the $\mathrm{C} 7$ atom participate in the intramolecular N16-H16A‥O18 and N16-H16B $\cdots \mathrm{N} 1$ hydrogen bond (Table 3; Fig. 2). In the packing, the molecules are linked into chains of rings along the $c$ axis (Fig. 3b). In these rings, four molecules of $N^{\prime}$-(4-aminophenylsulfonyl)-4-chloropicolinimidamide are linked via

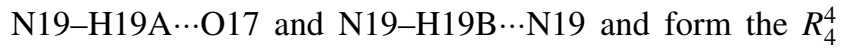
(20) hydrogen bond ring motif (Fig. 3a). The parallel lying chains of rings are connected through the N16H16A...O17 hydrogen bond and form columns along the $b$ axis (Table 3; Fig. 3a). In the crystal lattice, these columns form a zipper-type supramolecular motif.

\section{Tuberculostatic activity}

The synthesized phenylsulfonyl- and 4-aminophenylsulfonyl-carboximidamides 1-13 were examined in vitro for their tuberculostatic activity against M. tuberculosis $\mathrm{H}_{37} \mathrm{Rv}$ 
Table 1 Crystal data and structure refinement for $N^{\prime}$-(4-aminophenylsulfonyl)-4-chloropicolinimidamide

\begin{tabular}{|c|c|}
\hline Empirical formula & $\mathrm{C}_{12} \mathrm{H}_{11} \mathrm{ClN}_{4} \mathrm{O}_{2} \mathrm{~S}$ \\
\hline Formula weight & 310.76 \\
\hline Temperature/K & $295(2)$ \\
\hline Wavelength/Å & Crystal system \\
\hline \multirow[t]{3}{*}{ Space group } & 0.71073 \\
\hline & Monoclinic \\
\hline & $P 2_{1} / \mathrm{c}$ \\
\hline \multicolumn{2}{|l|}{ Unit cell dimensions } \\
\hline$a / \AA$ & $14.6885(7)$ \\
\hline$b / \AA$ & $5.7930(3)$ \\
\hline$c / \AA$ & $16.0421(9)$ \\
\hline$\beta /^{\circ}$ & $97.530(5)$ \\
\hline$V / \AA^{3}$ & $1353.25(12)$ \\
\hline$Z$ & 4 \\
\hline$D_{\text {calc }} / \mathrm{Mg} \mathrm{m}^{-3}$ & 1.525 \\
\hline Absorption coefficient $/ \mathrm{mm}^{-1}$ & 0.443 \\
\hline$F(000)$ & 640 \\
\hline Crystal size/mm & $0.35 \times 0.15 \times 0.10$ \\
\hline$\Theta$ Range for data collection $/^{\circ}$ & $3.08-25.00$ \\
\hline Limiting indices & $\begin{array}{c}-14 \leq h \leq 17,-6 \leq k \leq 6, \\
-19 \leq l \leq 13\end{array}$ \\
\hline Reflections collected/unique & $8,544 / 2,373\left(R_{\mathrm{int}}=0.0375\right)$ \\
\hline Completeness $2 \Theta=50.0 / \%$ & 99.8 \\
\hline Refinement method & Full-matrix least-squares on $F^{2}$ \\
\hline Data/restraints/parameters & $2,373 / 0 / 181$ \\
\hline Goodness-of-fit on $F^{2}$ & 1.006 \\
\hline \multirow[t]{2}{*}{ Final $R$ indices $[I>2 \sigma(I)]$} & $R_{1}=0.0406$ \\
\hline & $w R_{2}=0.1013$ \\
\hline \multirow[t]{2}{*}{$R$ indices (all data) } & $R_{1}=0.0626$ \\
\hline & $w R_{2}=0.1060$ \\
\hline Largest diff. peak and hole/e $\AA^{-3}$ & 0.369 and -0.345 \\
\hline
\end{tabular}

strain and two "wild" strains isolated from tuberculosis patients: one (Spec. 210) resistant to $p$-aminosalicylic acid (PAS), isonicotinic acid hydrazide (INH), etambutol (ETB), and rifampicine (RFP), and the other (Spec. 192) fully sensitive to the administered tuberculostatics (Table 4).

Investigations were performed by a classical test-tube method of successive dilution in Youmans' modification of Proskauer and Beck's liquid medium containing $10 \%$ of bovine serum $[33,34]$. Bacterial suspensions were prepared from 14-day-old cultures of slowly growing strains and from 48-h-old cultures of saprophytic strains [35, 36]. Solutions of the compounds in ethylene glycol were tested. Stock solutions contained $10 \mathrm{mg}$ of compounds in $1 \mathrm{~cm}^{3}$. Dilutions (in geometric progression) were prepared in Youmans' medium. The medium containing no investigated substances and containing isoniazid (INH) as a
Table 2 Selected bond lengths, valence angles, and torsion angles for $N^{\prime}$-(4-aminophenylsulfonyl)-4-

chloropicolinimidamide

\begin{tabular}{lr}
\hline Bond lengths/A & \\
N1-C2 & $1.331(3)$ \\
N1-C6 & $1.338(5)$ \\
C2-C7 & $1.496(3)$ \\
C4-C120 & $1.724(4)$ \\
C5-C6 & $1.355(5)$ \\
C7-N16 & $1.308(3)$ \\
C7-N8 & $1.311(3)$ \\
N8-S9 & $1.626(2)$ \\
S9-C10 & $1.742(2)$ \\
C13-N19 & $1.369(3)$ \\
Valence angles/ ${ }^{\circ}$ & \\
C2-N1-C6 & $116.1(3)$ \\
N8-C7-C2 & $115.9(2)$ \\
C7-N8-S9 & $120.8(2)$ \\
N8-S9-C10 & $104.6(1)$ \\
Torsion angles/ ${ }^{\circ}$ & \\
N1-C2-C7-N8 & $164.6(2)$ \\
C3-C2-C7-N8 & $-16.0(4)$ \\
C2-C7-N8-S9 & $178.1(2)$ \\
C7-N8-S9-C10 & $-87.8(2)$ \\
N8-S9-C10-C11 & $108.4(2)$ \\
\hline
\end{tabular}

Table 3 Hydrogen bonds for $N^{\prime}$-(4-aminophenylsulfonyl)-4-chloropicolinimidamide with distances $(\mathrm{d} / \AA): \quad \mathrm{d}(\mathrm{D} \cdots \mathrm{A})<\mathrm{R}(\mathrm{D})+$ $\mathrm{R}(\mathrm{A})+0.50 \AA ; \quad \mathrm{d}(\mathrm{H} \cdots \mathrm{A})<\mathrm{R}(\mathrm{H})+\mathrm{R}(\mathrm{A})-0.12 \AA$ and angle $^{\circ}$ $(<)<\mathrm{D}-\mathrm{H} \cdots \mathrm{A}>100.0^{\circ}$

\begin{tabular}{llllll}
\hline $\mathrm{D}-\mathrm{H}$ & $\mathrm{A}$ & $\mathrm{d}(\mathrm{D}-\mathrm{H})$ & $\mathrm{d}(\mathrm{H} \cdot \mathrm{A})$ & $<\mathrm{D}-\mathrm{H} \cdots \mathrm{A}$ & $\mathrm{d}(\mathrm{D} \cdot \mathrm{A})$ \\
\hline $\mathrm{N} 16-\mathrm{H} 16 \mathrm{~A}$ & $\mathrm{O}^{\mathrm{a}}$ & 0.86 & 2.15 & $2.758(3)$ & 128 \\
$\mathrm{~N} 16-\mathrm{H} 16 \mathrm{~B}$ & $\mathrm{N1}^{\mathrm{a}}$ & 0.86 & 2.26 & $2.630(3)$ & 105 \\
$\mathrm{~N} 16-\mathrm{H} 16 \mathrm{~A}$ & $\mathrm{O}^{\mathrm{b}}$ & 0.86 & 2.37 & $2.988(3)$ & 129 \\
$\mathrm{~N} 19-\mathrm{H} 19 \mathrm{~A}$ & $\mathrm{O}^{\mathrm{c}}$ & 0.86 & 2.31 & $3.035(3)$ & 142 \\
$\mathrm{~N} 19-\mathrm{H} 19 \mathrm{~B}$ & $\mathrm{~N}^{\mathrm{d}} 9^{\mathrm{d}}$ & 0.86 & 2.58 & $3.424(3)$ & 167 \\
\hline
\end{tabular}

${ }^{a}$ Intramolecular $\mathrm{H}$ bond

Symmetry codes: ${ }^{\mathrm{b}} x, y-1, z ;{ }^{\mathrm{c}} x, 1 / 2-y, z-1 / 2 ;{ }^{\mathrm{d}}-x, y-1 / 2,1 / 2-z$

reference drug were used for comparison. Incubation was performed at a temperature of $37^{\circ} \mathrm{C}$. The $M I C$ values were determined as minimum concentration inhibiting the growth of tested tuberculous strains in relation to the probe with no tested compound. The influence of the compound on the growth of bacteria at a certain concentration, 3.1, $6.2,12.5,25,50$, and $100 \mu \mathrm{g} / \mathrm{cm}^{3}$, was evaluated.

The study showed that the newly synthesized sulfonylcarboximidamides 1-13 exhibited very low tuberculostatic activity. Minimumal inhibitory concentration $(M I C)$ values for all the tested compounds ranged from 25 to $100 \mu \mathrm{g} /$ $\mathrm{cm}^{3}$. No significant differences in compounds' activity against the sensitive strain 192 and the resistant strain 210 

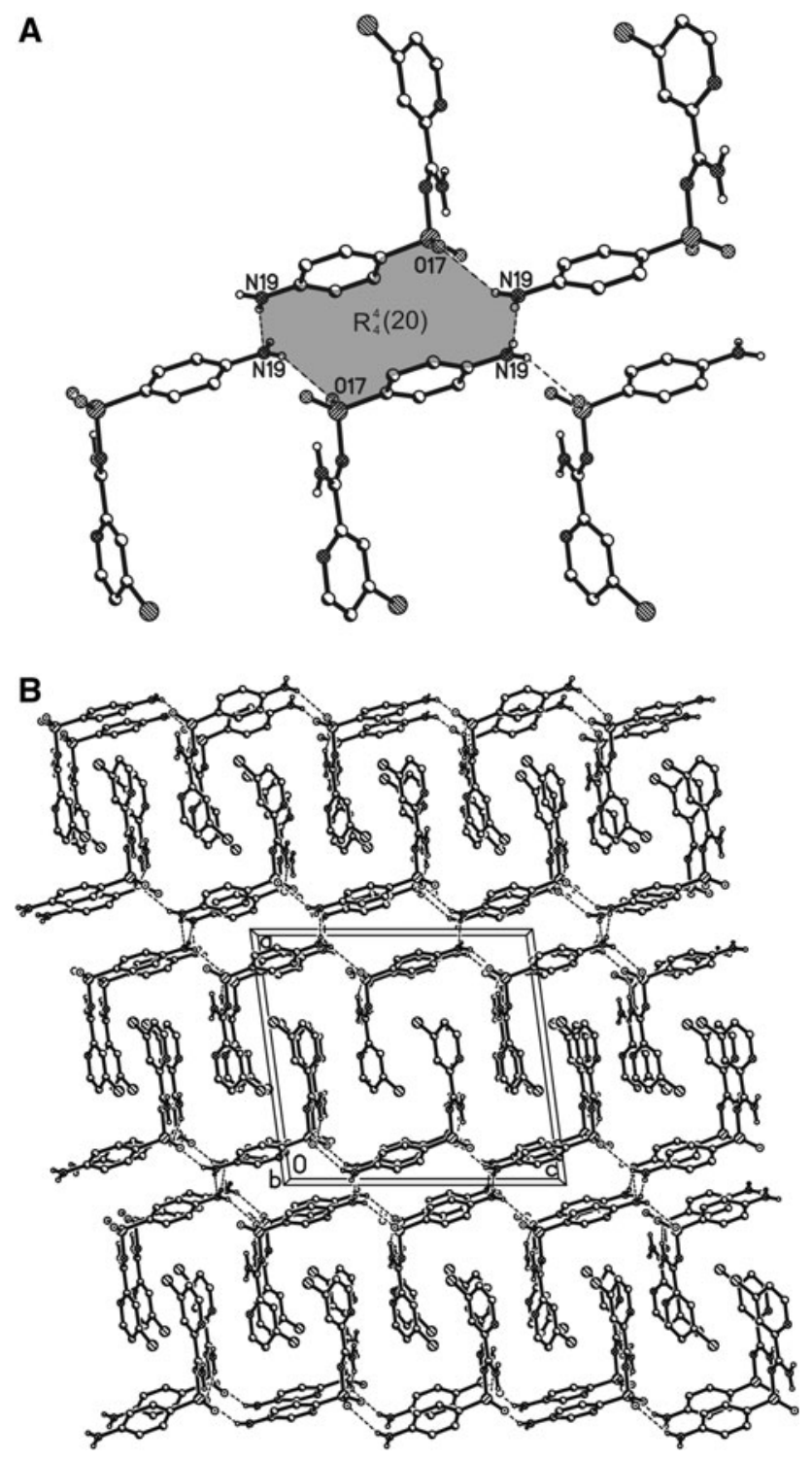

Fig. 3 The $R_{4}^{4}$ (20) hydrogen bond motif (a) and the arrangement of the molecules in the crystal structure of $N^{\prime}$-(4-aminophenylsulfonyl)4-chloropicolinimidamide viewed along $b$ axis (b). Dashed lines: $\mathrm{N}-\mathrm{H} \cdots \mathrm{O}$ and $\mathrm{N}-\mathrm{H} \cdots \mathrm{N}$ interactions. $\mathrm{H}$ atoms not involved in interactions omitted

have been observed. Isoniazid, the reference tuberculostatic, exhibited much higher activity with the MIC value $0.5-1.1 \mu \mathrm{g} / \mathrm{cm}^{3}$. These results classify the compounds tested as practically inactive against $M$. tuberculosis.

\section{Antibacterial and antifungal activities}

Antibacterial and antifungal activities of newly synthesized compounds were also examined. In the study of antibacterial activity three recommended reference strains $S$. aureus ATCC 25923, E. coli ATCC 25922, and P. aeruginosae ATCC 27853 were used [37]. Antifungal activity was determined with use of two strains: C. albicans ATCC 90028 and
C. parapsilosis ATCC 22019 [38]. The susceptibility of the microorganisms to the agents was determined by the broth microdilution assay according to the procedures outlined by the National Committee for Clinical Laboratory Standards $[37,38]$. The stock solutions of the agents were prepared by dissolving the chemicals in DMSO. The final concentration of the agents in $200 \mathrm{~mm}^{3}$ of Mueller-Hinton broth (for bacterial strains) or in RPMI 1,640 (for fungi) ranged over 0.125 $256 \mu \mathrm{g} / \mathrm{cm}^{3}$.

In order to prepare the bacterial suspension, overnight culture of bacteria in $3 \%$ Triptic soy broth was diluted in sterile saline to the final concentration of approximately $10^{7} \mathrm{CFU} / \mathrm{cm}^{3}$. Aliquots $\left(10 \mathrm{~mm}^{3}\right)$ of bacterial suspension were added to each agent solution. The $M I C$ was defined as the lowest concentration of the agent that completely inhibited growth of the bacteria after $18 \mathrm{~h}$ incubation at $35{ }^{\circ} \mathrm{C}$.

Inocula of candida strains were prepared by suspension of five colonies picked from $24 \mathrm{~h}$ old cultures on Saburaud agar in sterile saline to the concentration of $10^{6}$ cells per $\mathrm{cm}^{3}$. The final concentration of the working suspension was approximately $10^{4}$ cells per $\mathrm{cm}^{3}$. Aliquots $\left(10 \mathrm{~mm}^{3}\right)$ of the suspension were added to each agar solution. The MIC was defined as the lowest concentration of the agent that completely inhibited growth of the fungi after $48 \mathrm{~h}$ incubation at $35{ }^{\circ} \mathrm{C}$. The final results were average values from two independent experiments.

The study showed no antibacterial and antifungal activity of the tested compounds. All of the synthesized sulfonylcarboximidamides 1-13 exhibited activity with $M I C>256 \mu \mathrm{g} / \mathrm{cm}^{3}$, which meant that those values did not fit standard test concentrations.

\section{Conclusion}

In conclusion, a series of novel sulfonyl-carboximidamides with different six-membered nitrogen heterocyclic systems were synthesized successfully in a reaction of heterocyclic methyl carbimidates with benzene sulfonamide and 4-aminobenzenesulfonamide. All these new compounds were confirmed by IR and NMR spectra as well as elemental analysis. The molecular structure of the obtained compounds was discussed. Compounds possessing heterocyclic systems with a nitrogen atom in the $\alpha$ position to the functional group showed a single-crystal structure different from expected and described for that chemical group in the literature. Antimicrobial activity of the synthesized compounds was evaluated against M. tuberculosis, S. aureus, E. coli, P. aeruginosae, C. albicans, and C. parapsilosis. Unfortunately, all of the studied compounds were practically inactive towards microbial strains tested. 
Table 4 Antimicrobial activity of newly synthesized sulfonylcarboximidamides 1-13
Minimum inhibitory concentrations for bacterial strains were determined by the two-fold serial dilution method for microdilution plates and for mycobacterial strains by twofold classical test-tube method of successive dilution

INH isoniazid

\begin{tabular}{|c|c|c|c|c|c|c|c|c|}
\hline \multirow[t]{3}{*}{ No. } & \multicolumn{8}{|c|}{$M I C / \mu \mathrm{g} / \mathrm{cm}^{3}$} \\
\hline & \multicolumn{3}{|c|}{ M. tuberculosis } & \multicolumn{3}{|c|}{ Bacterial strains } & \multicolumn{2}{|c|}{ Fungal strains } \\
\hline & $\mathrm{H}_{37} \mathrm{Rv}$ & 192 & 210 & S. aureus & E. coli & P. aeruginosae & C. albicans & C. parapsilosis \\
\hline 1 & 100 & 50 & 50 & $>256$ & $>256$ & $>256$ & $>256$ & $>256$ \\
\hline 2 & 100 & 50 & 50 & $>256$ & $>256$ & $>256$ & $>256$ & $>256$ \\
\hline 3 & 50 & 50 & 50 & $>256$ & $>256$ & $>256$ & $>256$ & $>256$ \\
\hline 4 & 50 & 50 & 50 & $>256$ & $>256$ & $>256$ & $>256$ & $>256$ \\
\hline 5 & 50 & 50 & 50 & $>256$ & $>256$ & $>256$ & $>256$ & $>256$ \\
\hline 6 & 50 & 50 & 50 & $>256$ & $>256$ & $>256$ & $>256$ & $>256$ \\
\hline 7 & 50 & 100 & 50 & $>256$ & $>256$ & $>256$ & $>256$ & $>256$ \\
\hline 8 & 100 & 100 & 50 & $>256$ & $>256$ & $>256$ & $>256$ & $>256$ \\
\hline 9 & 50 & 50 & 50 & $>256$ & $>256$ & $>256$ & $>256$ & $>256$ \\
\hline 10 & 100 & 50 & 50 & $>256$ & $>256$ & $>256$ & $>256$ & $>256$ \\
\hline 11 & 50 & 50 & 50 & $>256$ & $>256$ & $>256$ & $>256$ & $>256$ \\
\hline 12 & 50 & 50 & 50 & $>256$ & $>256$ & $>256$ & $>256$ & $>256$ \\
\hline 13 & 50 & 50 & 25 & $>256$ & $>256$ & $>256$ & $>256$ & $>256$ \\
\hline INH & 0.5 & 0.5 & 1.1 & - & - & - & - & - \\
\hline
\end{tabular}

\section{Experimental}

All materials and solvents were of analytical reagent grade. Thin-layer chromatography was performed on Merck silica gel $60 \mathrm{~F}_{254}$ plates and visualized with UV. The results of elemental analyses $(\mathrm{C}, \mathrm{H}, \mathrm{N})$ for all obtained compounds were in agreement with calculated values within the range of $\pm 0.3 \%$. ${ }^{1} \mathrm{H}$ NMR spectra in $\mathrm{CDCl}_{3}$ or DMSO- $d_{6}$ were recorded on Varian Unity Plus $(500 \mathrm{MHz})$ and Varian Gemini (200 MHz) instruments (Varian, Palo Alto, CA). Infrared spectra were determined as $\mathrm{KBr}$ pellets of the solids on a Satellite FT-IR spectrophotometer (Mattson Instruments, Madison, WI). Melting points were determined with a Boethius apparatus (Franz Küstner Nachf. KG, Dresden, Germany). Methyl pyrazine-2-carbimidate and methyl 6-methoxypyrazine-2-carbimidate required for further syntheses were obtained according to the method described earlier by Foks and co-workers [22, 23].

\section{General method for the synthesis of sulfonyl- carboximidamides $\mathbf{1 - 8}$}

The respective carbonitrile $(1 \mathrm{mmol})$ and $0.4 \mathrm{~cm}^{3}$ ( $2 \mathrm{mmol}$ ) of DBU were refluxed in $10 \mathrm{~cm}^{3}$ of methanol for $0.5 \mathrm{~h}$. Then $0.8 \mathrm{mmol}$ of benzene sulfonamide or 4-aminobenzenesulfonamide was added. The mixture was refluxed for another $3 \mathrm{~h}$. Then methanol was evaporated in vacuo, and $30 \mathrm{~cm}^{3}$ of water was added to the residue. The precipitate of the product was filtered off, dried, and purified by recrystallization from a suitable solvent.
$N^{\prime}-\left(\right.$ Phenylsulfonyl)picolinimidamide $\left(1, \mathrm{C}_{12} \mathrm{H}_{11} \mathrm{~N}_{3} \mathrm{O}_{2} \mathrm{~S}\right)$ Recrystallization from ethanol afforded $138 \mathrm{mg}(66 \%) \mathbf{1}$. M.p.: $165-166{ }^{\circ} \mathrm{C} ; I R(\mathrm{KBr}): \bar{v}=3,432,3,320(v \mathrm{~N}-\mathrm{H})$, 1,613, 1,538 $(v \mathrm{C}=\mathrm{C}), 1,280,1,147\left(v \mathrm{SO}_{2}\right), 757(\gamma \mathrm{C}-\mathrm{H})$, $688(\gamma \mathrm{N}-\mathrm{H}), 589,557 \mathrm{~cm}^{-1} ;{ }^{1} \mathrm{H}$ NMR $(200 \mathrm{MHz}$, $\left.\mathrm{CDCl}_{3}\right): \delta=7.43-7.59\left(\mathrm{~m}, 4 \mathrm{H}, 3 \mathrm{H} \mathrm{Ph}\right.$ and $1 \mathrm{H} \mathrm{NH}+\mathrm{D}_{2} \mathrm{O}$ exchangeable), $7.82(\mathrm{~m}, 1 \mathrm{H}$, pyridine $), 8.02(\mathrm{~m}, 2 \mathrm{H}, \mathrm{Ph})$, $8.28\left(\mathrm{~m}, 2 \mathrm{H}\right.$, pyridine), 8.33 (brs, $1 \mathrm{H}, \mathrm{NH}+\mathrm{D}_{2} \mathrm{O}$ exchangeable), $8.58\left(\mathrm{~m}, 1 \mathrm{H}\right.$, pyridine) $\mathrm{ppm} ;{ }^{13} \mathrm{C} \mathrm{NMR}$ $\left(50 \mathrm{MHz}, \mathrm{CDCl}_{3}\right): \delta=123.10(\mathrm{C}-3), 126.38\left(\mathrm{C}-2^{\prime}, \mathrm{C}-6^{\prime}\right)$, 127.82 (C-5), $129.32\left(\mathrm{C}-3^{\prime}, \mathrm{C}^{-} 5^{\prime}\right), 132.71\left(\mathrm{C}-4^{\prime}\right), 138.40$ (C-4), $142.39\left({\mathrm{C}-1^{\prime}}^{\prime}\right), 148.71$ (C-6), 149.20 (C-2), 159.20 $(\mathrm{C}=\mathrm{N}) \mathrm{ppm}$.

$N^{\prime}$-(4-Aminophenylsulfonyl)picolinimidamide

$\left(2, \mathrm{C}_{12} \mathrm{H}_{12} \mathrm{~N}_{4} \mathrm{O}_{2} \mathrm{~S}\right)$

Recrystallization from dioxane afforded $124 \mathrm{mg}(56 \%)$ 2. M.p.: 202-205 ${ }^{\circ} \mathrm{C} ; I R(\mathrm{KBr}): \bar{v}=3,435,3,400,3,323$, 3,253 ( $v \mathrm{~N}-\mathrm{H}), 1,610,1,588(v \mathrm{C}=\mathrm{C}), 1,271,1,144(v$ $\left.\mathrm{SO}_{2}\right), 1,091(\delta \mathrm{C}-\mathrm{H}), 821(\gamma \mathrm{C}-\mathrm{H}), 566(\gamma \mathrm{N}-\mathrm{H}) \mathrm{cm}^{-1}$; ${ }^{1} \mathrm{H} \quad \mathrm{NMR} \quad\left(200 \mathrm{MHz}, \mathrm{CDCl}_{3}\right): \delta=5.94 \quad(\mathrm{~s}, \quad 2 \mathrm{H}$, $\mathrm{NH}_{2}+\mathrm{D}_{2} \mathrm{O}$ exchangeable), $6.52(\mathrm{~d}, 2 \mathrm{H}, \quad \mathrm{Ph}$, $J=8.6 \mathrm{~Hz}), 7.57$ (d, 2H, Ph, $J=8.8 \mathrm{~Hz}), 7.63-7.67$ (m, 1H, pyridine), 7.93-8.11 (m, 3H, $2 \mathrm{H}$ pyridine and $1 \mathrm{H}$ $\mathrm{NH}+\mathrm{D}_{2} \mathrm{O}$ exchangeable $), 8.67(\mathrm{~d}, 1 \mathrm{H}$, pyridine, $J=4.8 \mathrm{~Hz}$ ), 8.84 (brs, $1 \mathrm{H}, \mathrm{NH}+\mathrm{D}_{2} \mathrm{O}$ exchangeable) ppm; ${ }^{13} \mathrm{C}$ NMR (50 MHz, DMSO- $\left.d_{6}\right): \delta=112.79$ $\left(\mathrm{C}-3^{\prime}, \mathrm{C}-5^{\prime}\right), 122.83$ (C-3), 127.57 (C-5), 128.38 (C-2', C$\left.6^{\prime}\right), 138.26\left(\mathrm{C}-4, \mathrm{C}-1^{\prime}\right), 149.08$ (C-6, C-4'), 152.93 (C-2), $158.04(\mathrm{C}=\mathrm{N}) \mathrm{ppm}$. 
$N^{\prime}$-(Phenylsulfonyl)nicotinimidamide $\left(3, \mathrm{C}_{12} \mathrm{H}_{11} \mathrm{~N}_{3} \mathrm{O}_{2} \mathrm{~S}\right)$

Recrystallization from dioxane-methanol mixture (1:1) afforded $98 \mathrm{mg}(47 \%)$ 3. M.p.: $176-178{ }^{\circ} \mathrm{C} ; I R(\mathrm{KBr})$ : $\bar{v}=3,439,3,322(v \mathrm{~N}-\mathrm{H}), 3,054(v \mathrm{C}-\mathrm{H}), 1,618,1,518(v$ $\mathrm{C}=\mathrm{C}), 1,274,1,164,1,149\left(v \mathrm{SO}_{2}\right), 825,789(\gamma \mathrm{C}-\mathrm{H})$, $583(\gamma \mathrm{N}-\mathrm{H}), 561 \mathrm{~cm}^{-1} ;{ }^{1} \mathrm{H}$ NMR $\left(200 \mathrm{MHz}\right.$, DMSO- $\left.d_{6}\right)$ : $\delta=7.47-7.66(\mathrm{~m}, 4 \mathrm{H}, \mathrm{Ph}), 7.94-7.98(\mathrm{~m}, 2 \mathrm{H}, \mathrm{Ph}), 8.16-$ $8.20\left(\mathrm{~m}, 1 \mathrm{H}\right.$, pyridine), $8.30-8.60$ (brs, $1 \mathrm{H}, \mathrm{NH}+\mathrm{D}_{2} \mathrm{O}$ exchangeable), 8.73-8.75 (m, 1H, pyridine), $8.98(\mathrm{~d}, 1 \mathrm{H}$, pyridine, $J=1.47 \mathrm{~Hz}$ ), $9.10-9.40$ (brs, $1 \mathrm{H}, \mathrm{NH}+\mathrm{D}_{2} \mathrm{O}$ exchangeable) ppm; ${ }^{13} \mathrm{C}$ NMR $\left(50 \mathrm{MHz}\right.$, DMSO- $\left.d_{6}\right)$ : $\delta=123.71 \quad(\mathrm{C}-4), 125.83 \quad\left(\mathrm{C}-2^{\prime}, \mathrm{C}^{\prime} 6^{\prime}\right), 126.40 \quad\left(\mathrm{C}-3^{\prime}\right.$, C-5'), 129.67 (C-3), 132.06 (C-4'), 132.56 (C-4), 135.99 $\left(\mathrm{C}-1^{\prime}\right), 148.96(\mathrm{C}-2), 153.05(\mathrm{C}-6), 161.39(\mathrm{C}=\mathrm{N}) \mathrm{ppm}$.

\section{$N^{\prime}$-(4-Aminophenylsulfonyl)nicotinimidamide}

$\left(4, \mathrm{C}_{12} \mathrm{H}_{12} \mathrm{~N}_{4} \mathrm{O}_{2} \mathrm{~S}\right)$

Recrystallization from dioxane-ethanol mixture (1:1) afforded $132 \mathrm{mg}(60 \%)$ 4. M.p.: $215-217^{\circ} \mathrm{C}$; IR (KBr): $\bar{v}=3,448,3,394,3,337,3,313,3,248(v \mathrm{~N}-\mathrm{H}), 2,923$, $2,851(v \mathrm{C}-\mathrm{H}), 1,643,1,612,1,591,1,528(v \mathrm{C}=\mathrm{C})$, 1,269, 1,141 $\left(v \mathrm{SO}_{2}\right), 1,089(\delta \mathrm{C}-\mathrm{H}), 786,698(\gamma \mathrm{C}-\mathrm{H}), 562$ $(\gamma \mathrm{N}-\mathrm{H}) \mathrm{cm}^{-1} ;{ }^{1} \mathrm{H}$ NMR $\left(200 \mathrm{MHz}, \mathrm{DMSO}-d_{6}\right): \delta=5.92$ (s, $2 \mathrm{H}, \mathrm{NH}_{2}+\mathrm{D}_{2} \mathrm{O}$ exchangeable), $6.58(\mathrm{~d}, 2 \mathrm{H}, \mathrm{Ph}$, $J=8.7 \mathrm{~Hz}), 7.46-7.52(\mathrm{~m}, 1 \mathrm{H}$, pyridine $), 7.58(\mathrm{~d}, 2 \mathrm{H}$, $\mathrm{Ph}, J=8.7 \mathrm{~Hz}), 8.12-8.18(\mathrm{~m}, 2 \mathrm{H}, 1 \mathrm{H}$ pyridine and $1 \mathrm{H}$ $\mathrm{NH}+\mathrm{D}_{2} \mathrm{O}$ exchangeable $), 8.70-8.73(\mathrm{~m}, 1 \mathrm{H}$, pyridine $)$, $8.95(\mathrm{~d}, 1 \mathrm{H}$, pyridine, $J=1.9 \mathrm{~Hz}), 9.02\left(\mathrm{~s}, 1 \mathrm{H}, \mathrm{NH}+\mathrm{D}_{2} \mathrm{O}\right.$ exchangeable) ppm; ${ }^{13} \mathrm{C}$ NMR (50 MHz, DMSO- $\left.d_{6}\right)$ : $\delta=112.76\left(\mathrm{C}-3^{\prime}, \mathrm{C}^{\prime} 5^{\prime}\right), 123.68$ (C-5), $127.81 \quad(\mathrm{C}-3)$, $128.39\left(\mathrm{C}-2^{\prime}, \mathrm{C}-6^{\prime}\right), 129.86\left(\mathrm{C}-1^{\prime}\right), 135.84(\mathrm{C}-2, \mathrm{C}-4)$, $148.85(\mathrm{C}-6), 152.82\left(\mathrm{C}-4^{\prime}\right), 160.13(\mathrm{C}=\mathrm{N}) \mathrm{ppm}$.

\section{$N^{\prime}$-(Phenylsulfonyl)isonicotinimidamide}

$\left(5, \mathrm{C}_{12} \mathrm{H}_{11} \mathrm{~N}_{3} \mathrm{O}_{2} \mathrm{~S}\right)$

Recrystallization from methanol-water mixture (1:1) afforded $111 \mathrm{mg}(53 \%)$ 5. M.p.: $155-156{ }^{\circ} \mathrm{C} ; \quad I R \quad(\mathrm{KBr})$ : $\bar{v}=3,379(v \mathrm{~N}-\mathrm{H}), 3,058,2,925(v \mathrm{C}-\mathrm{H}), 1,644,1,530$ $(v \mathrm{C}=\mathrm{C}), 1,281,1,142\left(v \mathrm{SO}_{2}\right), 1,086(\delta \mathrm{C}-\mathrm{H}), 843(\gamma \mathrm{C}-$ $\mathrm{H}), 589(\gamma \mathrm{N}-\mathrm{H}), 556 \mathrm{~cm}^{-1} ;{ }^{1} \mathrm{H}$ NMR (200 MHz, DMSO$\left.d_{6}\right): \delta=7.54-7.76(\mathrm{~m}, 5 \mathrm{H}, \mathrm{Ph}), 7.96(\mathrm{~d}, 2 \mathrm{H}$, pyridine, $J=6.6 \mathrm{~Hz}), 8.49\left(\mathrm{~s}, 1 \mathrm{H}, \mathrm{NH}+\mathrm{D}_{2} \mathrm{O}\right.$ exchangeable $), 8.71$ (d, $2 \mathrm{H}$, pyridine, $J=5 \mathrm{~Hz}$ ), 9.30 (brs, $1 \mathrm{H}, \mathrm{NH}+\mathrm{D}_{2} \mathrm{O}$ exchangeable) ppm; ${ }^{13} \mathrm{C}$ NMR $(50 \mathrm{MHz}$, DMSO-d 6 ): $\delta=121.90$ (C-3, C-5), 126.42 (C-2', C-6'), 129.27 (C-3', C-5'), 132.65 (C-4'), 141.26 (C-4), $142.41\left(\mathrm{C}-1^{\prime}\right), 150.52$ $(\mathrm{C}-2, \mathrm{C}-6), 161.23(\mathrm{C}=\mathrm{N}) \mathrm{ppm}$.

\section{$N^{\prime}$-(4-Aminophenylsulfonyl)isonicotinimidamide}

$\left(6, \mathrm{C}_{12} \mathrm{H}_{12} \mathrm{~N}_{4} \mathrm{O}_{2} \mathrm{~S}\right)$

Recrystallization from dioxane-ethanol mixture (1:1) afforded $152 \mathrm{mg}(69 \%)$ 6. M.p.: $226-229{ }^{\circ} \mathrm{C} ; \mathrm{IR}(\mathrm{KBr})$ : $\bar{v}=3,441,3,357,3,242(v \mathrm{~N}-\mathrm{H}), 2,957,2,849(v \mathrm{C}-\mathrm{H})$, $1,644,1,596,1,527(v \mathrm{C}=\mathrm{C}), 1,276,1,136\left(v \mathrm{SO}_{2}\right), 1,084$ $(\delta \mathrm{C}-\mathrm{H}), 828(\gamma \mathrm{C}-\mathrm{H}), 556(\gamma \mathrm{N}-\mathrm{H}) \mathrm{cm}^{-1} ;{ }^{1} \mathrm{H}$ NMR $\left(500 \mathrm{MHz}\right.$, DMSO- $\left.d_{6}\right): \delta=5.96\left(\mathrm{~s}, 2 \mathrm{H}, \mathrm{NH}_{2}+\mathrm{D}_{2} \mathrm{O}\right.$ exchangeable), $6.59(\mathrm{~d}, 2 \mathrm{H}, J=8.8 \mathrm{~Hz}), 7.74(\mathrm{~d}, 2 \mathrm{H}$, pyridine, $J=5.9 \mathrm{~Hz}), \quad 8.25$ (brs, $1 \mathrm{H}, \quad \mathrm{NH}+\mathrm{D}_{2} \mathrm{O}$ exchangeable), $8.71(\mathrm{~d}, 2 \mathrm{H}$, pyridine, $J=5.9 \mathrm{~Hz}), 9.10$ (brs, $1 \mathrm{H}, \mathrm{NH}+\mathrm{D}_{2} \mathrm{O}$ exchangeable) ppm; ${ }^{13} \mathrm{C}$ NMR $\left(50 \mathrm{MHz}, \mathrm{DMSO}-d_{6}\right): \delta=112.78\left({\mathrm{C}-3^{\prime}}^{\prime}, \mathrm{C}^{-} 5^{\prime}\right), 121.95$ (C-3, C-5), 126.43 (C-2', C-6'), $129.87\left(\mathrm{C}-1^{\prime}\right), 141.36$ (C-4), $150.48 \quad(\mathrm{C}-2, \quad \mathrm{C}-6), \quad 152.85 \quad\left(\mathrm{C}-4^{\prime}\right), \quad 161.28$ $(\mathrm{C}=\mathrm{N}) \mathrm{ppm}$.

\section{$N^{\prime}$-(Phenylsulfonyl)pyrimidine-2-carboximidamide}

$\left(7, \mathrm{C}_{11} \mathrm{H}_{10} \mathrm{~N}_{4} \mathrm{O}_{2} \mathrm{~S}\right)$

Recrystallization from dioxane afforded $107 \mathrm{mg}(51 \%) 7$. M.p.: $206-208{ }^{\circ} \mathrm{C} ; I R(\mathrm{KBr}): \bar{v}=3,396,3,330(v \mathrm{~N}-\mathrm{H})$, $1,621,1,554(v \mathrm{C}=\mathrm{C}), 1,280,1,151\left(v \mathrm{SO}_{2}\right), 833,790,689$ $(\gamma \mathrm{C}-\mathrm{H}), 590(\gamma \mathrm{N}-\mathrm{H}), 501 \mathrm{~cm}^{-1} ;{ }^{1} \mathrm{H}$ NMR $(200 \mathrm{MHz}$, DMSO- $\left.d_{6}\right): \delta=7.54-7.73(4 \mathrm{H}, 3 \mathrm{H} \mathrm{Ph}$ and $1 \mathrm{H}$ pyrimidine), $7.91(\mathrm{~d}, 2 \mathrm{H}, \mathrm{Ph}, J=8.2 \mathrm{~Hz}), 8.21$ (brs, $1 \mathrm{H}$, $\mathrm{NH}+\mathrm{D}_{2} \mathrm{O}$ exchangeable), 8.53 (brs, $1 \mathrm{H}, \mathrm{NH}+\mathrm{D}_{2} \mathrm{O}$ exchangeable), $8.96(\mathrm{~d}, 2 \mathrm{H}$, pyrimidine, $J=4.6 \mathrm{~Hz}) \mathrm{ppm}$; ${ }^{13} \mathrm{C}$ NMR (50 MHz, DMSO- $d_{6}$ ): $\delta=123.58$ (C-5), 126.50 (C-2', C-6'), 129.27 (C-3', C-5'), 132.68 (C-4'), 142.39 (C$\left.1^{\prime}\right), 158.11(\mathrm{C}-4, \mathrm{C}-6), 158.53(\mathrm{C}-2), 159.24(\mathrm{C}=\mathrm{N}) \mathrm{ppm}$.

$N^{\prime}$-(4-Aminophenylsulfonyl)pyrimidine-2-carboximidamide $\left(8, \mathrm{C}_{11} \mathrm{H}_{11} \mathrm{~N}_{5} \mathrm{O}_{2} \mathrm{~S}\right)$

Recrystallization from ethylene glycol-methanol mixture (1:1) afforded $175 \mathrm{mg}(79 \%)$ 8. M.p.: $259-261{ }^{\circ} \mathrm{C}$; IR $(\mathrm{KBr}): \bar{v}=3,380,3,330,3,237(v \mathrm{~N}-\mathrm{H}), 1,621,1,592$, $1,562,1,503,1,391(v \mathrm{C}=\mathrm{C}), 1,268,1,142\left(v \mathrm{SO}_{2}\right), 830$, $787(\gamma \mathrm{C}-\mathrm{H}), 678,578(\gamma \mathrm{N}-\mathrm{H}), 546 \mathrm{~cm}^{-1} ;{ }^{1} \mathrm{H}$ NMR $\left(500 \mathrm{MHz}\right.$, DMSO- $\left.d_{6}\right): \delta=5.95\left(\mathrm{~s}, 2 \mathrm{H}, \mathrm{NH}_{2}+\mathrm{D}_{2} \mathrm{O}\right.$ exchangeable), $6.52(\mathrm{~d}, 2 \mathrm{H}, \mathrm{Ph}, J=8.8 \mathrm{~Hz}), 7.54(\mathrm{~d}, 2 \mathrm{H}$, $\mathrm{Ph}, J=8.8 \mathrm{~Hz}$ ), $7.57(\mathrm{t}, 1 \mathrm{H}$, pyrimidine, $J=4.8 \mathrm{~Hz}$ ), 8.20 (brs, $1 \mathrm{H}, \mathrm{NH}+\mathrm{D}_{2} \mathrm{O}$ exchangeable), 8.87 (brs, $1 \mathrm{H}$, $\mathrm{NH}+\mathrm{D}_{2} \mathrm{O}$ exchangeable $), 8.94(\mathrm{~d}, 2 \mathrm{H}$, pyrimidine, $J=4.8 \mathrm{~Hz}) \mathrm{ppm} ;{ }^{13} \mathrm{C}$ NMR $\left(50 \mathrm{MHz}, \quad\right.$ DMSO- $\left.d_{6}\right)$ : $\delta=112.76\left(\mathrm{C}-3^{\prime}, \mathrm{C}^{\prime} 5^{\prime}\right), 123.50(\mathrm{C}-5), 127.51 \quad\left(\mathrm{C}-1^{\prime}\right)$, $128.54\left(\mathrm{C}-2^{\prime}, \mathrm{C}-6^{\prime}\right), 152.96\left(\mathrm{C}-4^{\prime}\right), 157.71$ (C-2), 158.09 $(\mathrm{C}-4, \mathrm{C}-6), 158.50(\mathrm{C}=\mathrm{N}) \mathrm{ppm}$.

$N^{\prime}$-[4-[N-[Amino(pyrimidin-2-yl)methylene]sulfamoyl]phenyl]acetamide $\left(\mathbf{9}, \mathrm{C}_{13} \mathrm{H}_{13} \mathrm{~N}_{5} \mathrm{O}_{3} \mathrm{~S}\right)$

Method A: the title compound was obtained according to the method described above for compounds 1-8 from $0.11 \mathrm{~cm}^{3}(1 \mathrm{mmol})$ of 2-cyanopyrimidine and $0.43 \mathrm{~g}$ (2 mmol) of $N$-(4-sulfamoylphenyl)acetamide affording $112 \mathrm{mg}(35 \%) 9$.

Method B: sulfonylcarboximidamide $8(0.28 \mathrm{~g}, 1 \mathrm{mmol})$ was refluxed for $0.5 \mathrm{~h}$ in a solution of $0.5 \mathrm{~cm}^{3}(5 \mathrm{mmol})$ of acetic anhydride in $5 \mathrm{~cm}^{3}$ of pyridine. Then pyridine was evaporated in vacuo, and $20 \mathrm{~g}$ of ice was added to the residue. The precipitate was filtered off, dried, and 
recrystallized from ethylene glycol to afford $268 \mathrm{mg}$ (84\%) 9.

M.p.: $253-254{ }^{\circ} \mathrm{C} ; I R(\mathrm{KBr}): \bar{v}=3,385,3,301(v \mathrm{~N}-\mathrm{H})$, $2,924,2,854(v \mathrm{C}-\mathrm{H}), 1,684(v \mathrm{C}=\mathrm{O}), 1,624,1,590$, $1,562,1,525,1,401(v \mathrm{C}=\mathrm{C}), 1,280,1,148\left(v \mathrm{SO}_{2}\right), 736(\gamma$ $\mathrm{C}-\mathrm{H}), 565(\gamma \mathrm{N}-\mathrm{H}) \mathrm{cm}^{-1} ;{ }^{1} \mathrm{H}$ NMR $(500 \mathrm{MHz}$, DMSO$\left.d_{6}\right): \delta=2.09\left(\mathrm{~s}, 3 \mathrm{H}, \mathrm{CH}_{3}\right), 7.72(\mathrm{t}, 1 \mathrm{H}$, pyrimidine, $J=4.8 \mathrm{~Hz}), 7.75(\mathrm{~d}, 2 \mathrm{H}, \mathrm{Ph}, J=8.8 \mathrm{~Hz}), 7.86(\mathrm{~d}, 2 \mathrm{H}, \mathrm{Ph}$, $J=8.8 \mathrm{~Hz}$ ), 8.42 (brs, $1 \mathrm{H}, \mathrm{NH}+\mathrm{D}_{2} \mathrm{O}$ exchangeable), $8.96(\mathrm{~d}, 2 \mathrm{H}$, pyrimidine, $J=4.1 \mathrm{~Hz}$ ), 9.08 (brs, $1 \mathrm{H}$, $\mathrm{NH}+\mathrm{D}_{2} \mathrm{O}$ exchangeable), $10.33\left(\mathrm{~s}, 1 \mathrm{H}, \mathrm{NH}+\mathrm{D}_{2} \mathrm{O}\right.$ exchangeable) ppm; ${ }^{13} \mathrm{C}$ NMR $\left(50 \mathrm{MHz}\right.$, DMSO- $\left.d_{6}\right)$ : $\delta=24.39 \quad\left(\mathrm{CH}_{3}\right), 118.73 \quad\left(\mathrm{C}-3^{\prime}, \mathrm{C}-5^{\prime}\right), 123.58 \quad(\mathrm{C}-5)$, $127.73\left(\mathrm{C}-2^{\prime}\right), 136.03\left(\mathrm{C}-1^{\prime}\right), 143.08\left(\mathrm{C}-4^{\prime}\right), 158.11(\mathrm{C}-4$, C-6), $158.46(\mathrm{C}-2), 158.80(\mathrm{C}=\mathrm{N}) \mathrm{ppm}$.

\section{General procedure for the synthesis} of sulfonylcarboximidamides 10-13

Methyl pyrazine-2-carbimidate or methyl 6-methoxypyrazine-2-carbimidate $(3 \mathrm{mmol})$ and the respective sulfonamide $(2.5 \mathrm{mmol})$ were refluxed in $5 \mathrm{~cm}^{3}$ of diglyme for $15 \mathrm{~min}$. After cooling down $20 \mathrm{~g}$ of ice was added to the mixture, and the precipitate of the product was filtered off, dried, and purified by recrystallization from a suitable solvent with activated carbon.

\section{$N^{\prime}$-(Phenylsulfonyl)pyrazine-2-carboximidamide}

$\left(\mathbf{1 0}, \mathrm{C}_{11} \mathrm{H}_{10} \mathrm{~N}_{4} \mathrm{O}_{2} \mathrm{~S}\right)$

Recrystallization from dioxane afforded $249 \mathrm{mg}$ (38\%) 10. M.p.: $\quad 218-219^{\circ} \mathrm{C} ; \quad I R \quad(\mathrm{KBr}): \quad \bar{v}=3,434, \quad 3,321$ $(v \mathrm{~N}-\mathrm{H}), 1,612,1,545(v \mathrm{C}=\mathrm{C}), 1,278,1,151\left(v \mathrm{SO}_{2}\right)$, $801,686(\gamma \mathrm{C}-\mathrm{H}), 590(\gamma \mathrm{N}-\mathrm{H}) \mathrm{cm}^{-1} ;{ }^{1} \mathrm{H}$ NMR $(200 \mathrm{MHz}$, DMSO- $\left.d_{6}\right): \delta=7.54-7.69(\mathrm{~m}, 3 \mathrm{H}, \mathrm{Ph}), 7.98(\mathrm{~d}, 2 \mathrm{H}, \mathrm{Ph}$, $J=7.3 \mathrm{~Hz}$ ), 8.44 (brs, $1 \mathrm{H}, \mathrm{NH}+\mathrm{D}_{2} \mathrm{O}$ exchangeable), 8.77 (s, $1 \mathrm{H}$, pyrazine), 8.90 (s, $1 \mathrm{H}$, pyrazine), 9.15 (brs, $1 \mathrm{H}$, $\mathrm{NH}+\mathrm{D}_{2} \mathrm{O}$ exchangeable), 9.23 (s, $1 \mathrm{H}$, pyrazine) ppm; ${ }^{13} \mathrm{C}$ NMR (50 MHz, DMSO- $\left.d_{6}\right): \delta=126.49\left(\mathrm{C}-2^{\prime}, \mathrm{C}-6^{\prime}\right)$, $129.35\left(\mathrm{C}-3^{\prime}, \mathrm{C}-5^{\prime}\right), 132.84\left(\mathrm{C}-4^{\prime}\right), 143.92(\mathrm{C}-2), 144.21$ $(\mathrm{C}-3, \mathrm{C}-5), 144.54\left(\mathrm{C}-1^{\prime}\right), 148.49(\mathrm{C}-6), 158.28(\mathrm{C}=\mathrm{N})$ ppm.

\section{$N^{\prime}$-(4-Aminophenylsulfonyl)pyrazine-2-carboximidamide} (11, $\left.\mathrm{C}_{11} \mathrm{H}_{11} \mathrm{~N}_{5} \mathrm{O}_{2} \mathrm{~S}\right)$

Recrystallization from dioxane afforded $381 \mathrm{mg}(55 \%) \mathbf{1 1 .}$ M.p.: $247-249{ }^{\circ} \mathrm{C} ; I R(\mathrm{KBr}): \bar{v}=3,431,3,394,3,320$, 3,252 $(v \mathrm{~N}-\mathrm{H}), 1,612,1,593(v \mathrm{C}=\mathrm{C}), 1,268,1,145(v$ $\left.\mathrm{SO}_{2}\right), 1,092(\delta \mathrm{C}-\mathrm{H}), 798(\gamma \mathrm{C}-\mathrm{H}), 567(\gamma \mathrm{N}-\mathrm{H}) \mathrm{cm}^{-1} ;{ }^{1} \mathrm{H}$ NMR $\left(200 \mathrm{MHz}, \mathrm{DMSO}-d_{6}\right): \delta=5.97\left(\mathrm{~s}, 2 \mathrm{H}, \mathrm{NH}_{2}+\mathrm{D}_{2} \mathrm{O}\right.$ exchangeable), 6.57 (d, 2H, Ph, $J=8.7 \mathrm{~Hz}), 7.59$ (d, 2H, $\mathrm{Ph}, J=8.7 \mathrm{~Hz}$ ), 8.14 (brs, $1 \mathrm{H}, \mathrm{NH}+\mathrm{D}_{2} \mathrm{O}$ exchangeable), $8.75(\mathrm{~d}, 1 \mathrm{H}$, pyrazine, $J=2.4 \mathrm{~Hz}), 8.89(\mathrm{~d}, 1 \mathrm{H}$, pyrazine, $J=2.4 \mathrm{~Hz}$ ), 8.92 (brs, $1 \mathrm{H}, \mathrm{NH}+\mathrm{D}_{2} \mathrm{O}$ exchangeable), 9.20 (s, $1 \mathrm{H}$, pyrazine) ppm; ${ }^{13} \mathrm{C}$ NMR $(50 \mathrm{MHz}$, DMSO- $\left.d_{6}\right): \delta=112.82\left(\mathrm{C}-3^{\prime}, \mathrm{C}-5^{\prime}\right), 128.53\left(\mathrm{C}-2^{\prime}, \mathrm{C}-6^{\prime}\right), 143.83$ (C-2), 144.02 (C-3, C-5), 144.78 (C-1'), 148.23 (C-6), $153.05\left(\mathrm{C}-4^{\prime}\right), 157.05(\mathrm{C}=\mathrm{N}) \mathrm{ppm}$.

$N^{\prime}$-(Phenylsulfonyl)-6-methoxypyrazine-2-carboximidamide $\left(\mathbf{1 2}, \mathrm{C}_{12} \mathrm{H}_{12} \mathrm{~N}_{4} \mathrm{O}_{3} \mathrm{~S}\right)$

Recrystallization from ethanol afforded $584 \mathrm{mg}(80 \%) \mathbf{1 2}$. M.p.: $156-157{ }^{\circ} \mathrm{C} ; I R(\mathrm{KBr}): \bar{v}=3,395,3,300(v \mathrm{~N}-\mathrm{H})$, $1,640,1,580,1,543(v \mathrm{C}=\mathrm{C}), 1,383(\delta \mathrm{C}-\mathrm{H}), 1,306,1,144$ $\left(v \mathrm{SO}_{2}\right), 1,008(\delta \mathrm{C}-\mathrm{H}), 803(\gamma \mathrm{C}-\mathrm{H}), 591(\gamma \mathrm{N}-\mathrm{H}) \mathrm{cm}^{-1}$; ${ }^{1} \mathrm{H}$ NMR $\left(200 \mathrm{MHz}\right.$, DMSO- $\left.d_{6}\right): \delta=4.03\left(\mathrm{~s}, 3 \mathrm{H}, \mathrm{OCH}_{3}\right)$, 7.53-7.65 (m, 3H, Ph), 8.00 (d, 2H, Ph, $J=7.6 \mathrm{~Hz}), 8.50$ (brs, $1 \mathrm{H}, \mathrm{NH}+\mathrm{D}_{2} \mathrm{O}$ exchangeable), $8.53(\mathrm{~s}, 1 \mathrm{H}$, pyrazine), $8.77\left(\mathrm{~s}, 1 \mathrm{H}\right.$, pyrazine), 9.02 (brs, $1 \mathrm{H}, \mathrm{NH}+\mathrm{D}_{2} \mathrm{O}$ exchangeable) ppm; ${ }^{13} \mathrm{C}$ NMR $\left(50 \mathrm{MHz}\right.$, DMSO- $\left.d_{6}\right)$ : $\delta=54.54\left(\mathrm{OCH}_{3}\right), 126.44\left(\mathrm{C}-2^{\prime}, \mathrm{C}-6^{\prime}\right), 129.33\left(\mathrm{C}-3^{\prime}\right.$, C-5'), 132.77 (C-3), 135.61 (C-4'), 139.67 (C-2), 140.88 $\left(\mathrm{C}-1^{\prime}\right), 142.24(\mathrm{C}-5), 158.27(\mathrm{C}-6), 159.01(\mathrm{C}=\mathrm{N}) \mathrm{ppm}$.

$N^{\prime}$-(4-Aminophenylsulfonyl)-6-methoxypyrazine-2-carboximidamide (13, $\left.\mathrm{C}_{12} \mathrm{H}_{13} \mathrm{~N}_{5} \mathrm{O}_{3} \mathrm{~S}\right)$

Recrystallization from methanol afforded $637 \mathrm{mg}(83 \%)$ 13. M.p.: $188-189{ }^{\circ} \mathrm{C}$; $I R(\mathrm{KBr}): \bar{v}=3,468,3,417,3,370$, $3,309,3,244(v \mathrm{~N}-\mathrm{H}), 1,634,1,584,1,545(v \mathrm{C}=\mathrm{C}), 1,379$ $(\delta \mathrm{C}-\mathrm{H}), 1,318,1,261,1,133\left(v \mathrm{SO}_{2}\right), 1,079(\delta \mathrm{C}-\mathrm{H}), 788$ $(\gamma \mathrm{C}-\mathrm{H}), 544(\gamma \mathrm{N}-\mathrm{H}) \mathrm{cm}^{-1}$; ${ }^{1} \mathrm{H}$ NMR $(200 \mathrm{MHz}$, DMSO$\left.d_{6}\right): \delta=4.02\left(\mathrm{~s}, 3 \mathrm{H}, \mathrm{OCH}_{3}\right), 5.95\left(\mathrm{~s}, 2 \mathrm{H}, \mathrm{NH}_{2}+\mathrm{D}_{2} \mathrm{O}\right.$ exchangeable), 6.59 (d, 2H, Ph, $J=8.8 \mathrm{~Hz}), 7.59$ (d, $2 \mathrm{H}$, $\mathrm{Ph}, J=8.5 \mathrm{~Hz}), 8.21\left(\mathrm{~s}, 1 \mathrm{H}, \mathrm{NH}+\mathrm{D}_{2} \mathrm{O}\right.$ exchangeable), $8.51(\mathrm{~s}, 1 \mathrm{H}$, pyrazine), $8.73(\mathrm{~s}, 1 \mathrm{H}$, pyrazine), $8.77(\mathrm{~s}, 1 \mathrm{H}$, $\mathrm{NH}+\mathrm{D}_{2} \mathrm{O}$ exchangeable) ppm; ${ }^{13} \mathrm{C}$ NMR $(50 \mathrm{MHz}$, DMSO- $\left.d_{6}\right): \quad \delta=54.52\left(\mathrm{OCH}_{3}\right), 112.76\left(\mathrm{C}-3^{\prime}, \mathrm{C}-5^{\prime}\right)$, 128.56 (C-2', C-6'), 132.74 (C-3), 139.84 (C-2), 141.76 $\left(\mathrm{C}-1^{\prime}\right), 142.27(\mathrm{C}-5), 158.29(\mathrm{C}-6), 157.88(\mathrm{C}=\mathrm{N}) \mathrm{ppm}$.

Crystal structure of $N^{\prime}$-(4-aminophenylsulfonyl)-4chloropicolinimidamide

Single crystals of $N^{\prime}$-(4-aminophenylsulfonyl)-4-chloropicolinimidamide suitable for X-ray diffraction were obtained from ethanol by slow evaporation of the solvent at room temperature. Good quality single-crystal specimens were selected for experiments at $T=295(2) \mathrm{K}$. They were mounted with epoxy glue at the tip of glass capillaries. Diffraction data were collected on an Oxford Diffraction Gemini R ULTRA Ruby CCD diffractometer with MoK $\alpha$ radiation $(\lambda=0.71073 \AA)$. The lattice parameters were obtained by least-squares fit to the optimized setting angles of the collected reflections by means of CrysAlis CCD [27]. Data were reduced by using CrysAlis RED [27] software with applying multi-scan absorption corrections (empirical absorption correction using spherical harmonics, implemented in SCALE3 ABSPACK scaling algorithm). The structural resolution procedure was made using the 
SHELXS-97 package solving the structures by direct methods and carrying out refinements by full-matrix leastsquares on $F^{2}$ using the SHELXL-97 program [28]. All H atoms bound with aromatic $\mathrm{C}$ atoms were placed geometrically and refined using a riding model with $\mathrm{C}-\mathrm{H}=0.93 \AA$ and $U_{\text {iso }}(\mathrm{H})=1.2 U_{\text {eq }}(\mathrm{C})$. All $\mathrm{H}$ atoms bound with $\mathrm{N}$ atoms were placed geometrically and refined using a riding model with $\mathrm{N}-\mathrm{H}=0.86 \AA$ and $U_{\text {iso }}(\mathrm{H})=1.5 U_{\text {eq }}(\mathrm{N})$. The $-\mathrm{NH}_{2}$ group containing the $\mathrm{N} 19$ atom was assumed to be planar-trigonal and coplanar with the mean plane of the benzene ring. The $-\mathrm{NH}_{2}$ group containing the N16 atom was assumed to be planar-trigonal and coplanar with the mean plane delineated by $\mathrm{C} 2, \mathrm{C} 7$, and N8 atoms. All interactions demonstrated were found by the PLATON program [29]. The programs used to prepare molecular graphics were: ORTEPII [30], PLUTO-78 [31], and Mercury [32]. Full crystallographic details, excluding structural features, have been deposited (deposition no. 849210) with the Cambridge Crystallographic Data Center. These data may be obtained, on request, from the Director, CCDC, 12 Union Road, Cambridge, CB2 1EZ, UK (Tel.: +44-1223-336408; Fax: +44-1223-336033; e-mail:deposit@ccdc.cam.ac.uk or http://www.ccdc.cam.ac.uk).

Open Access This article is distributed under the terms of the Creative Commons Attribution License which permits any use, distribution, and reproduction in any medium, provided the original author(s) and the source are credited.

\section{References}

1. Gomez-Lus R, Clavel A, Castillo J, Seral C, Rubio C (2000) Int J Antimicrob Agents 16:335

2. Appelbaum PC (2007) Clin Infect Dis $45: \mathrm{S} 165$

3. Prasad R, Kapoor K (2004) Int Rev Cytol 242:215

4. Gandhi NR, Nunn P, Dheda K, Schaaf HS, Zignol M, van Soolingen D, Jensen P, Bayona J (2010) Lancet 375:1830

5. Wells CD, Cegielski JP, Nelson LJ, Laserson KF, Holtz TH, Finlay A, Castro KG, Weyer K (2007) J Infect Dis 196:S68

6. Norrby SR, Nord CE, Finch R (2005) Lancet Infect Dis 5:115

7. Supuran CT (2008) Nat Rev Drug Disc 7:168

8. Isik S, Kockar F, Aydin M, Arslan O, Guler OO, Innocenti A, Scozzafava A, Supuran CT (2009) Bioorg Med Chem 17:1158

9. Gawin R, De Clercq E, Naesens L, Koszytkowska-Stawińska M (2008) Bioorg Med Chem 16:8379

10. Bouissane L, Kazzouli SE, Léonce S, Pfeiffer B, Rakib EM, Khouili M, Guillaument G (2006) Bioorg Med Chem 14:1078
11. Weber A, Casini A, Heine A, Kuhn D, Supuran CT, Scozzafava A, Kiebe G (2004) J Med Chem 47:550

12. Panico A, Vicini P, Incert M, Cardile V, Gentile B, Ronsisvalle G (2002) Il Farmaco 57:671

13. Sienkiewicz P, Bielawski K, Bielawska A, Palka J (2005) Environ Toxicol Pharmacol 20:118

14. Sielecki TM, Liu J, Mousa SA, Racanelli AL, Hausner EA, Waxler RR, Olson RE (2001) Bioorg Med Chem Lett 11:2201

15. Echevarria A, Santos LH, Miller J, Mahmood N (1996) Bioorg Med Chem Lett 6:1901

16. Bedi PMS, Mahajan MP, Kapoor VK (2004) Bioorg Med Chem Lett 14:3821

17. Greenidge PA, Carlsson B, Bladh L-G, Gillner M (1998) J Med Chem 41:2503

18. Dubina VL, Shebitchenko LN, Pedan VP, Yukhno AG, Skripets VI (1982) Russ J Org Chem 18:691

19. Rossi E, Stradi R, Benedusi A (1987) Tetrahedron 43:4785

20. Fujisawa T, Mizuno C (1952) Yakugaku Zasshi 72:694

21. Northey EH, Pierce AE, Kartesz DJ (1942) J Am Chem Soc 64:2763

22. Foks H, Janowiec M (1979) Acta Polon Pharm 36:155

23. Foks H, Manowska W (1976) Pol J Pharmacol Parm 28:49

24. Bogdanowicz A, Foks H, Kędzia A, Kwapisz E, Zwolska Z, Augustynowicz-Kopeć E (2009) Heterocycles 78:2217

25. Shilcrat SC, Mokhallati MK, Fortunak JMD, Pridgen LN (1997) J Org Chem 62:8449

26. Hopkins KT, Wilson WD, Bender BC, McCurdy DR, Hall JE (1998) J Med Chem 41:3872

27. Oxford Diffraction (2008) CrysAlis CCD and CrysAlis RED. Yarnton, England

28. Sheldrick GM (2008) Acta Cryst A64:112

29. Spek AL (2009) Acta Cryst D65:148

30. Johnson CK (1976) ORTEP II. Report ORNL-5138. Oak Ridge National Laboratory, Oak Ridge

31. Mortherwell S (1978) Clegg S PLUTO-78 program for drawing and molecular structure. University of Cambridge, UK

32. Macrae CF, Bruno IJ, Chisholm JA, Edgington PR, McCabe P, Pidcock E, Rodriguez-Monge L, Taylor R, van de Streek J, Wood PAJ (2008) Appl Cryst 41:466

33. Youmans GP (1947) Am Rev Tuberc 56:376

34. Youmans GP, Youmans AS (1949) J Bacteriol 58:247

35. Atlas RM, Singler JW (1995) Media for clinical microbiology. CRC Press, Boca Raton, p 313

36. Foks H, Buraczewska M, Manowska W, Sawlewicz J (1971) Dissert Pharm Pharmacol 23:49

37. National Committee for Clinical Laboratory Standards (1993) Methods for dilution antimicrobial susceptibility test for bacteria that grow aerobically. 3rd edn. Approved standard NCCLS document M7-A3, No 24, vol 13, NCCLS, Villanova

38. National Committee for Clinical Laboratory Standards (1993) Reference method for broth dilution antifungal susceptibility testing of yeast. Proposed Standard Document M27-P, Vol 13, No 24, NCCLS, Villanova 